\title{
CLL bei Älteren: Ibrutinib in der Erstlinie bald schon Standard?
}

Ibrutinib ist eine Option für ältere Patienten mit chronischer lymphatischer Leukämie (CLL). In einer Phase-III-Studie war eine Therapie mit dem Brutontyrosinkinase(BTK)-Hemmer dem alten Standard Chlorambucil überlegen.

hlorambucil war bis vor kurzem der Standard für ältere, häufig multimorbide Patienten mit CLL. Erst die Zugabe neuer, überwiegend zielgerichteter Substanzen, z. B. gegen CD20 gerichtete Antikörper, hat die Prognose verbessert. Auch Ibrutinib erwies sich in dieser Indikation als gut wirksam. Bisher hat es eine Zulassung für rezidivierte Formen. Nun wurde der BTK-Hemmer als Monotherapeutikum für die CLL-Erstlinientherapie bei Älteren überprüft. In der multizentrischen Phase-III-Studie RESONATE-2 erhielten 269 therapienaive CLL-Patienten
(> 65 Jahre) randomisiert Ibrutinib oder Chlorambucil. Primärer Endpunkt war das progressionsfreie Überleben (PFS). Nach median 18,4 Monaten lag es unter Chlorambucil bei median 18,9 Monaten, unter Ibrutinib war der Median noch nicht erreicht. Das Risiko für Progress oder Tod war in der Interventionsgruppe um $84 \%$ reduziert (Hazard Ratio [HR] 0,$16 ; p<0,001$ ). Auch die Rate für das Gesamtüberleben war unter dem BTK-Inhibitor höher: Nach 2 Jahren lebten noch 98 vs. $85 \%$ der Patienten (HR 0,16; $\mathrm{p}=0,001$ ). Mit 86 vs. $35 \%$ ergab sich zudem ein deut- licher Vorteil bei der Gesamtansprechrate zugunsten des BTK-Inhibitors $(\mathrm{p}<0,001)$. Auch bei der dauerhaften $\mathrm{Zu}$ nahme von Hämoglobin und Blutplättchen zeigte sich Ibrutinib dem Klassiker Chlorambucil überlegen - bei akzeptabler Verträglichkeit.

Fazit: Ältere Patienten mit therapienaiver CLL haben unter dem oralen BTKInhibitor Ibrutinib ein im Vergleich zu Chlorambucil signifikant längeres PFS und Gesamtüberleben, Hämoglobinund Blutplättchenspiegel steigen deutlich an.

Barbara Kreutzkamp

Burger JA et al. Ibrutinib as initial therapy for patients with chronic lymphocytic leukemia. N Engl J Med. 2015;373:2425-37.

\section{CLL: Ofatumumab-Erhaltung verlängert PFS}

\section{Beim follikulären Lymphom verlängert eine Erhaltungstherapie mit dem CD20-Antikörper Rituximab das PFS klinisch relevant. Bisher war die Frage der Erhaltungstherapie bei der CLL nicht geklärt. Nun gibt es neue Daten hierzu.}

$\mathrm{n}$ der Phase-III-Studie PROLONG wurde bei 474 Patienten untersucht, ob eine Erhaltungstherapie mit dem gegen CD20 gerichteten Antikörper Ofatumumab die Prognose von CLL-Patienten in kompletter oder partieller Remission nach einer Zweit- oder Drittlinientherapie weiter verbessern kann. Primärer Endpunkt

war das untersucherbestimmte progressionsfreie Überleben (PFS).

Zunächst erhielten die Patienten 1-mal $300 \mathrm{mg}$ Ofatumumab, nach einer Woche wurde die Dosis auf 1.000 mg i.v. gesteigert und danach alle 8 Wochen für bis $\mathrm{zu}$ 2 Jahre in dieser Dosierung gegeben. Die Patienten im Kontrollarm wurden nur

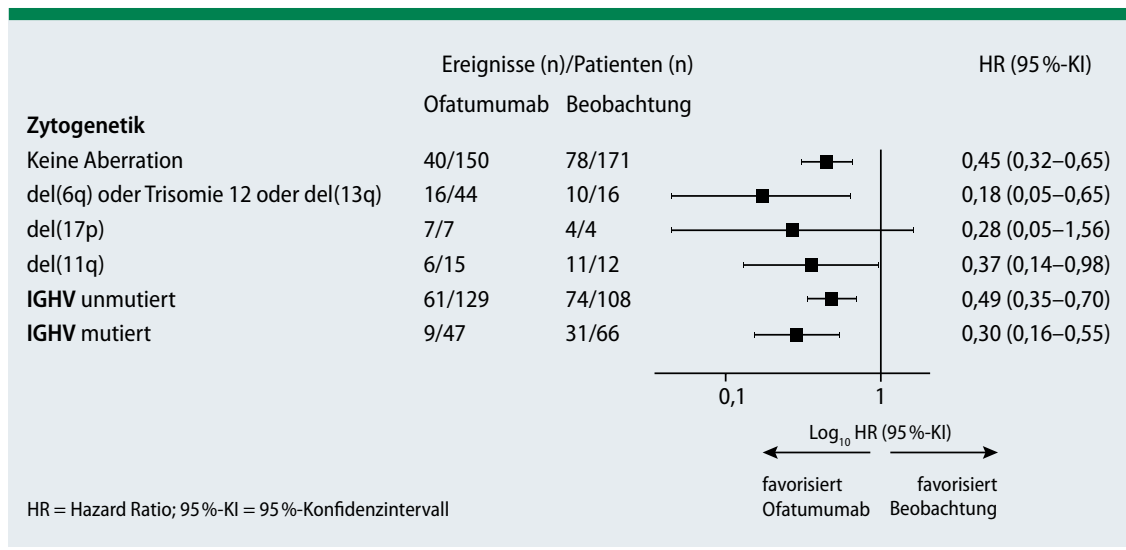

Abb. 1: Die PFS-Verlängerung war unabhängig von Zytogenetik und Mutationsstatus. beobachtet. Nach median 19,1 Monaten waren unter Ofatumumab 78 Ereignisse (74-mal Progress, 4 Todesfälle) gegenüber 120 (116-mal Progress, 4 Todesfälle) im Kontrollarm aufgetreten. Das untersucherbestimmte PFS war unter Ofatumumab mit median 29,4 gegenüber 15,2 Monaten im Kontrollarm signifikant und klinisch relevant länger (Hazard Ratio $0,50 ; \mathrm{p}<0,0001)$. Auch das durch ein unabhängiges Review-Komitee bestimmte PFS war unter Ofatumumab länger $(30,4$ vs. 14,8 Monate). Hinsichtlich des Überlebens fand sich kein Unterschied. In allen Subgruppen (berücksichtigt wurden etwa Alter, Remissionsstatus zu Studienbeginn, Binet-Stadium, Zytogenetik, $\beta_{2}$ Mikroglobulin) verlängerte Ofatumumab das PFS (Abb. 1).

Fazit: Diese Resultate sind ein wichtiger Baustein für die Entwicklung optimaler Erhaltungstherapien, vor allem, weil heutzutage neue, zielgerichtete Therapien eingesetzt und häufig bis zum Progress gegeben werden. Brigitte Schalhorn

van Oers MH et al. on behalf of the PROLONG study investigators. Ofatumumab maintenance versus observation in relapsed chronic lymphocytic leukaemia (PROLONG): an open-label, multicentre, randomised phase 3 study. Lancet Oncol. 2015;16(13):1370-9. 